\title{
EFFECTS OF PREDATOR DIET BREADTH ON STABILITY OF SIZE SPECTRA
}

\author{
M. J. PLANK ${ }^{1}$ \\ (Received 13 October, 2011; revised 11 January, 2012)
}

\begin{abstract}
Many marine ecosystems have the remarkable property that the abundance of organisms of a given body size is approximately proportional to the inverse square of that size. Size-structured models have been developed for which this "invariance-of-biomass" state is an equilibrium solution. These models are built on the coupling of predator growth to prey abundance, where prey suitability is determined by a size-based function referred to as a feeding kernel. In this paper, the local stability of the equilibrium state is investigated in a limiting case where predators only consume prey of a preferred size. In this special case, it is shown analytically that the equilibrium state is always unstable. It is concluded that some degree of diet breadth, in terms of the range of prey sizes consumed by a predator, is an essential prerequisite for the invariance-of-biomass state to be stable, as widely observed in the field.
\end{abstract}

2010 Mathematics subject classification: 92D40.

Keywords and phrases: body mass, ecosystem dynamics, McKendrick-von Foerster equation, predator, prey, size-structured model.

\section{Introduction}

A remarkable pattern observed in marine ecosystems is that if the organisms contained in some volume of the sea are binned into logarithmic intervals of body mass, the total biomass in each of these intervals varies relatively little over orders of magnitude of body size [8, 20, 31, 32]. For instance, the total biomass of organisms with body mass between $1 \mathrm{~g}$ and $10 \mathrm{~g}$ is roughly the same as that of organisms with body mass between $10 \mathrm{~g}$ and $100 \mathrm{~g}$, and so on. This pattern can be equivalently expressed in terms of a "size spectrum", in which abundance density $\phi(w)$ (number of organisms per unit body mass per unit volume of water) is related to body mass $w$ via a power-law relationship:

$$
\phi(w) \propto w^{-\gamma}
$$

\footnotetext{
${ }^{1}$ Department of Mathematics and Statistics, University of Canterbury, Christchurch, New Zealand; e-mail: michael.plank@ canterbury.ac.nz.

(c) Australian Mathematical Society 2012, Serial-fee code 1446-1811/2012 \$16.00
} 
where the exponent $\gamma$ is approximately $2[16,25,28,30]$. This empirical pattern has motivated the development of theoretical models that focus on body size, rather than species identity, as the most important factor in predator-prey interactions in marine ecosystems $[2,4,22,33]$. This contrasts with the more traditional perspective of food webs structured by species [1]. The size-based approach allows organisms to grow through orders of magnitude of body size as they feed, changing their prey as they do so.

Most models of size spectra use the well-known McKendrick-von Foerster equation $[23,36]$ to describe the dynamics of a community with size-dependent growth and mortality rates. Growth rates are typically determined by the abundance of prey; the range of suitable prey sizes is controlled by a feeding kernel. Datta et al. [12] showed that the McKendrick-von Foerster equation is actually a firstorder approximation to a more general jump-growth equation, which is based on the assumption that predators increase in size by a finite amount as a result of consuming a prey item. The jump-growth model accounts for variations between individuals more accurately than the McKendrick-von Foerster equation. For instance, under the McKendrick-von Foerster equation, a cohort of fish initially all of identical size will always remain of identical size; under the jump-growth equation, the cohort will become distributed over a range of sizes as it grows [12].

The existence of a power-law steady state of the form described by equation (1.1) has been demonstrated in models based on the McKendrick-von Foerster equation [2, 4] and the jump-growth equation [10, 12]. However, the factors affecting the stability of this steady state have been less well studied. Law et al. [21] used numerical solutions to show that the steady state of the McKendrick-von Foerster equation can be unstable, in the sense that the system does not return to the steady state following a small perturbation. Datta et al. [13] studied the stability of the jumpgrowth equation and obtained an integral expression for the eigenvalues of small perturbations to the steady state. Numerical evaluation of these eigenvalues showed that, as with the McKendrick-von Foerster equation, the steady state can be stable or unstable depending on model parameters. Capitan and Delius [10] investigated stability in a model incorporating terms for metabolic losses and reproduction and also obtained integral expressions for the eigenvalues. These and other studies of stability (for example, in a pelagic-benthic system [7]) have used numerical techniques either to solve the time-dependent system or to calculate eigenvalues of an appropriate operator.

All models of size spectra make some assumption about the range of prey sizes consumed by a predator. Possibly the simplest assumption is the one made by Camacho and Solé [9] that a predator indiscriminately consumes any prey smaller than itself. However, empirical data indicate that mean prey size is approximately proportional to predator size $[11,35]$. The mean predator-to-prey mass ratio (PPMR) is approximately 100 [17, 18], with some spread about this mean value [5, 19]. This is intuitively reasonable: most predators are limited by gape size and are therefore unable to consume prey that are too close to their own size [29]; conversely, the 
amount of energy gained from prey smaller than a certain size will not justify the energy expenditure required to catch them [34].

Benoît and Rochet [4] modelled the distribution of prey sizes targeted by a predator using a feeding kernel, which was assumed to be a gamma distribution of PPMR. More recently, most models have used a log-normal distribution for the feeding kernel $[2,6,15,21,26]$. The mean and standard deviation of this log-normal distribution are model parameters that respectively define the mean PPMR and the "diet breadth", that is, the range of prey sizes consumed by a predator.

In this paper, a limiting case of the log-normal feeding kernel is investigated: a Dirac delta function. This is useful as it allows us to obtain analytical results that would be difficult or impossible to obtain in the more general setting. These analytical results are valuable as they help to eliminate the possibility of inaccuracy in numerical results. The limiting case also has a clear biological meaning, which is that predators only eat prey with exactly the preferred size, that is, they have zero diet breadth. An analytical argument is presented showing that the power-law steady state of the jump-growth equation is always unstable in this case. This confirms previous numerical results showing that a narrow range of prey sizes can cause a switch to instability [13, 24]. It also shows definitively that some variability in prey size selection is an essential prerequisite for a stable ecosystem.

\section{Model}

The model considered here is the jump-growth model developed by Datta et al. [12]. The central assumption of the model is that when a predator of body mass $w$ consumes a prey item of body mass $w^{\prime}$, the prey dies and the predator's mass increases to $w+K w^{\prime}$, where $K$ is a constant representing the proportion of prey biomass that is converted to predator biomass. The state of the system at time $t$ is described by the abundance density $\phi(w, t)$, where $\phi(w, t) \delta w$ is the number of organisms per unit volume of water, whose mass lies in the range $[w, w+\delta w]$, in the limit $\delta w \rightarrow 0$. For notational simplicity, the argument $t$ is omitted in the following and the abundance density is denoted simply by $\phi(w)$.

There are three main ways in which the abundance density of organisms of mass $w$ can change: (i) an organism of mass $w$ eats a prey item and grows to a larger mass; (ii) an organism of mass $w$ is eaten by a larger predator; (iii) an organism grows to mass $w$ as a result of eating a prey item. These three processes, illustrated in Figure 1, give rise to three integral terms in the jump-growth equation developed by Datta et al. [12]:

$$
\begin{aligned}
\frac{\partial}{\partial t} \phi(w)=\int( & -\overbrace{T\left(w, w^{\prime}\right) \phi(w) \phi\left(w^{\prime}\right)}^{\text {growth to larger mass }}-\overbrace{T\left(w^{\prime}, w\right) \phi\left(w^{\prime}\right) \phi(w)}^{\text {death due to predation }} \\
& +\underbrace{T\left(w-K w^{\prime}, w^{\prime}\right) \phi\left(w-K w^{\prime}\right) \phi\left(w^{\prime}\right)}_{\text {growth from smaller mass }}) d w^{\prime}-\underbrace{\mu \phi(w)}_{\text {intrinsic mortality }} .
\end{aligned}
$$




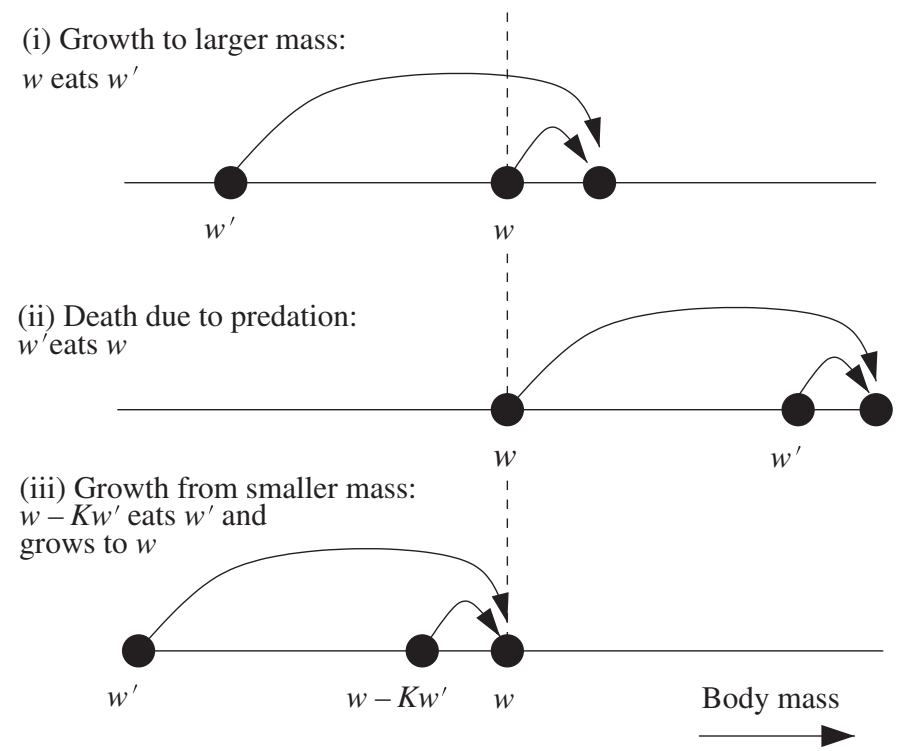

FiguRE 1. Predator-prey interactions corresponding to the three integral terms in the jump-growth equation (2.1). Interactions (i) and (ii) reduce the abundance of organisms of mass $w$; interaction (iii) increases the abundance of organisms of mass $w$.

The integral kernel $T\left(w, w^{\prime}\right)$ in equation (2.1) describes the feeding rate of predators of mass $w$ on prey of mass $w^{\prime}$. This is a combination of the volumetric search rate of a predator of mass $w$ and the selectivity of a predator of mass $w$ for prey of mass $w^{\prime}$. Following Andersen and Beyer [2] and Benoît and Rochet [4], we model the former as an increasing power-law function of predator mass $w$, and the latter as a function of the PPMR $w / w^{\prime}$ :

$$
T\left(w, w^{\prime}\right)=A w^{\alpha} s\left(w / w^{\prime}\right),
$$

where $A$ and $\alpha$ are positive constants. The function $s$ is referred to as the feeding kernel. The final term in equation (2.1) represents intrinsic mortality (that is, death from causes other than predation), which is assumed to be independent of mass.

It is convenient to introduce the new variables

$$
x=\ln \left(w / w_{0}\right), \quad u(x)=w_{0} e^{x} \phi(w),
$$

where $w_{0}$ is some arbitrary weight. In these new variables, and with a suitable change of integration variable, equation (2.1) becomes

$$
\begin{array}{r}
\frac{\partial}{\partial t} u(x)=A w_{0}^{\alpha} e^{\alpha x} \int s\left(e^{r}\right)\left[-u(x) u(x-r)-e^{\alpha r} u(x) u(x+r)\right. \\
\left.+e^{-\alpha \psi(r)} u(x-\psi(r)) u(x-r-\psi(r))\right] d r-\mu u(x),
\end{array}
$$

where $e^{\psi(r)}=1+K e^{-r}$ is the factor by which a predator's mass increases as a result of consuming a prey item with a PPMR of $e^{r}$. 


\section{Steady state}

This section provides an outline of previous work in which the steady state of equation (2.3) has been determined and solutions obtained for the linearized system close to this steady state. Full details are given by Datta et al. [13].

In the new variables defined by equation (2.2), a power-law size spectrum of the form (1.1) becomes

$$
u_{s}(x)=u_{0} e^{(1-\gamma) x} \text {. }
$$

This is a steady state of equation (2.3) provided that

$$
e^{(-\gamma+\alpha+1) x} \int s\left(e^{r}\right)\left[-e^{(\gamma-1) r}-e^{(-\gamma+\alpha+1) r}+e^{(\gamma-1) r+(2 \gamma-\alpha-2) \psi(r)}\right] d r-\eta=0,
$$

where $\eta=\mu /\left(A w_{0}^{\alpha} u_{0}\right)$ is a dimensionless parameter describing the strength of intrinsic mortality relative to predation. If there is some intrinsic mortality (that is, $\eta>0$ ) then equation (3.2) implies that $\gamma=\alpha+1$ and

$$
\int s\left(e^{r}\right)\left[-e^{\alpha r}-1+e^{\alpha(r+\psi(r))}\right] d r-\eta=0 .
$$

The integral in equation (3.3) is monotonically increasing with respect to $\alpha$. Hence, for given values of $\eta$ and $K$ and a given choice of feeding kernel, this equation determines a unique value for the search rate exponent $\alpha$ (and hence the steady state exponent $\gamma$ ). This imposes a constraint on the ecological parameters that must be satisfied in order for equation (2.1) to have a power-law steady state [10, 24]. This is an additional model assumption that may not always be ecologically realistic [13]. However, parameter values satisfying this constraint (see Section 4) are within ranges estimated from empirical data [5, 37]. In light of the widespread evidence for powerlaw size spectra $[16,25,28,30]$, it is assumed from now on that this constraint is satisfied.

The stability of the steady state (3.1) may be investigated by seeking time-dependent solutions to equation (2.3), linearized about the steady state. These solutions take the form of plane-wave perturbations with respect to the body mass variable $x$ :

$$
u(x, t)=u_{0} e^{(1-\gamma) x}\left(1+\epsilon e^{i k x+\lambda(k) t}\right),
$$

where $\epsilon>0$ is small. Importantly, a plane-wave perturbation with wavenumber $k$ will grow in amplitude if the real part of the eigenvalue $\lambda(k)$ is positive and decay if it is negative. Hence the real parts of the eigenvalues $\lambda(k)$ determine the stability of the steady state. By substituting equation (3.4) into equation (2.3) and linearizing (neglecting terms of order $\epsilon^{2}$ and higher), Datta et al. [13] obtained the following expression for the eigenvalues of these plane-wave perturbations:

$$
\lambda(k)=\int s\left(e^{r}\right)\left(-e^{\alpha r}-e^{i k r}+e^{\alpha r+(\alpha-i k) \psi(r)}\right)\left(1+e^{-i k r}\right) d r-\eta
$$




\section{Results}

Combining equation (3.5) with (3.3) shows that $\lambda(k)$ tends to $\eta$ as the wavenumber $k$ tends to zero. Hence, if there is some intrinsic mortality $(\eta>0)$ then the steady state is always unstable. However, arbitrarily small wavenumbers correspond to arbitrarily long wavelength perturbations to the steady state. In reality, size spectra consist of a finite range of body masses, and perturbations with a wavelength longer than this range are not realistic solutions. Indeed, the solution corresponding to $k=0$ corresponds ecologically to a uniform change in abundance density across all body masses. In general there are other factors that preclude such solutions. These may include a limited supply of food for the smallest members of the community, or senescent, nonpredation mortality that tends to prevent organisms growing past a certain maximum size $[15,24]$. It is therefore the eigenvalues corresponding to wavenumbers above a minimum threshold value, determined by the range of body masses in the ecosystem, that determine the stability of the steady state in real size spectra.

Many recent models have assumed that the feeding kernel is a Gaussian function of the logarithm of the PPMR $[2,6]$ :

$$
s\left(e^{r}\right)=\frac{1}{\sqrt{2 \pi} \sigma} \exp \left(-\frac{(r-\beta)^{2}}{2 \sigma^{2}}\right),
$$

where $\beta$ is the preferred PPMR and $\sigma$ is the diet breadth parameter. In the remainder of this section, we investigate the limiting case of $\sigma \rightarrow 0$, which gives a Dirac delta feeding kernel:

$$
s\left(e^{r}\right)=\delta(r-\beta) .
$$

This represents a community where predators feed exclusively on prey with body mass a factor of $e^{\beta}$ smaller than their own.

It is now shown that there are eigenvalues with positive real part for arbitrarily large values of the wavenumber $k$. The steady-state condition (3.3), in the case where the feeding kernel is a Dirac delta function, is

$$
-e^{\alpha \beta}-1+e^{\alpha(\beta+\psi)}=\eta,
$$

where $\psi=\ln \left(1+K e^{-\beta}\right)$. Now using equations (4.2) and (4.3) in the eigenvalue equation (3.5) and taking the real part gives

$$
\operatorname{Re}(\lambda)=e^{\alpha(\beta+\psi)}(-\cos (k \beta)+\cos (k \psi)+\cos (k(\beta+\psi))-1)+\eta \cos (k \beta) .
$$

Consider first the case where $\eta>0$ (that is, where there is some intrinsic mortality). Choosing $k \psi=2 n \pi$ for some positive integer $n$ gives

$$
\operatorname{Re}(\lambda)=\eta \cos \left(\frac{2 n \pi \beta}{\psi}\right) .
$$

Regardless of the value of $\beta / \psi$, this will be positive for some choice of $n>0$, including arbitrarily large choices of $n$ (which correspond to arbitrarily large wavenumbers $k$ ). 
Now consider the case where $\eta=0$ (no intrinsic mortality). Equation (4.4) shows that when $k=k^{*}=\pi / \beta, \operatorname{Re}(\lambda)$ is zero and

$$
\frac{d}{d k} \operatorname{Re}(\lambda)=e^{\alpha(\beta+\psi)} \beta \sin \left(\frac{\pi \psi}{\beta}\right) .
$$

This is nonzero unless $\psi=n \beta$ for some positive integer $n$. However $\psi=\ln \left(1+K e^{-\beta}\right)$ is less than $\beta$ unless

$$
e^{\beta} \leq \frac{1+\sqrt{1+4 K}}{2} .
$$

Since the biomass conversion efficiency $K$ must be less than 1, this would require the mean PPMR $e^{\beta}$ to be less than 1.6. This is unrealistic: PPMRs observed in the field are typically of the order $100[17,19]$. Hence at $k=k^{*}, \operatorname{Re}(\lambda)$ is zero and has nonzero derivative with respect to $k$. Therefore $\operatorname{Re}(\lambda)$ must be strictly positive for $k$ in some neighbourhood of $k^{*}$. Since $\operatorname{Re}(\lambda)$ is a periodic function of $k$ with period $2 \pi /(\beta+\psi)$, there are always infinitely many values of $k$ for which $\operatorname{Re}(\lambda)$ is positive, including arbitrarily large values.

The above argument shows that the steady state (3.1), where $\alpha$ is constrained via equation (3.3), is always unstable. The caveat to this is that, in the special case where $\eta=0$, it is seen from equation (3.2) that a power-law steady state exists for any value of $\alpha$. If $\alpha$ does not satisfy equation (3.3) then the linearized solutions are not plane-wave perturbations and no analytical expression for the eigenvalues is available [13, 24]. Nevertheless, including some intrinsic mortality in the model is realistic [14], in which case the instability result always holds.

Figure 2 shows the real part of the eigenvalues against wavenumber $k$ for a Dirac delta feeding kernel (given by equation (4.4)) and for a Gaussian feeding kernel (calculated according to equations (3.5) and (4.1)). The Dirac delta feeding kernel is a limiting case of the Gaussian feeding kernel as $\sigma \rightarrow 0$ and this is illustrated by the graphs. When $\sigma$ is relatively small (Figure 2, top panel), the eigenvalues for the Gaussian kernel closely track those for the delta kernel for small wavenumbers (long wavelength perturbations), but decay onto a monotonically decreasing curve for larger wavenumbers. When $\sigma$ is larger (Figure 2, bottom panel), this decay occurs sooner, that is, for smaller wavenumbers. This allows for the possibility of a stable steady state $(\operatorname{Re}(\lambda(k))<0$ for all $k)$ if $\sigma$ is sufficiently large, as found in numerical calculations [24]. Figure 2 confirms that, in contrast to the Gaussian kernel, the eigenvalues for the delta kernel are always of positive real part for strictly positive values of $k$.

\section{Discussion}

This paper has focused on the jump-growth model for the dynamics of a community with size-dependent predation and growth. The jump-growth model is a generalization of the well-known McKendrick-von Foerster model for a size-structured population. The jump-growth model incorporates higher-order, diffusion-like effects, for example 

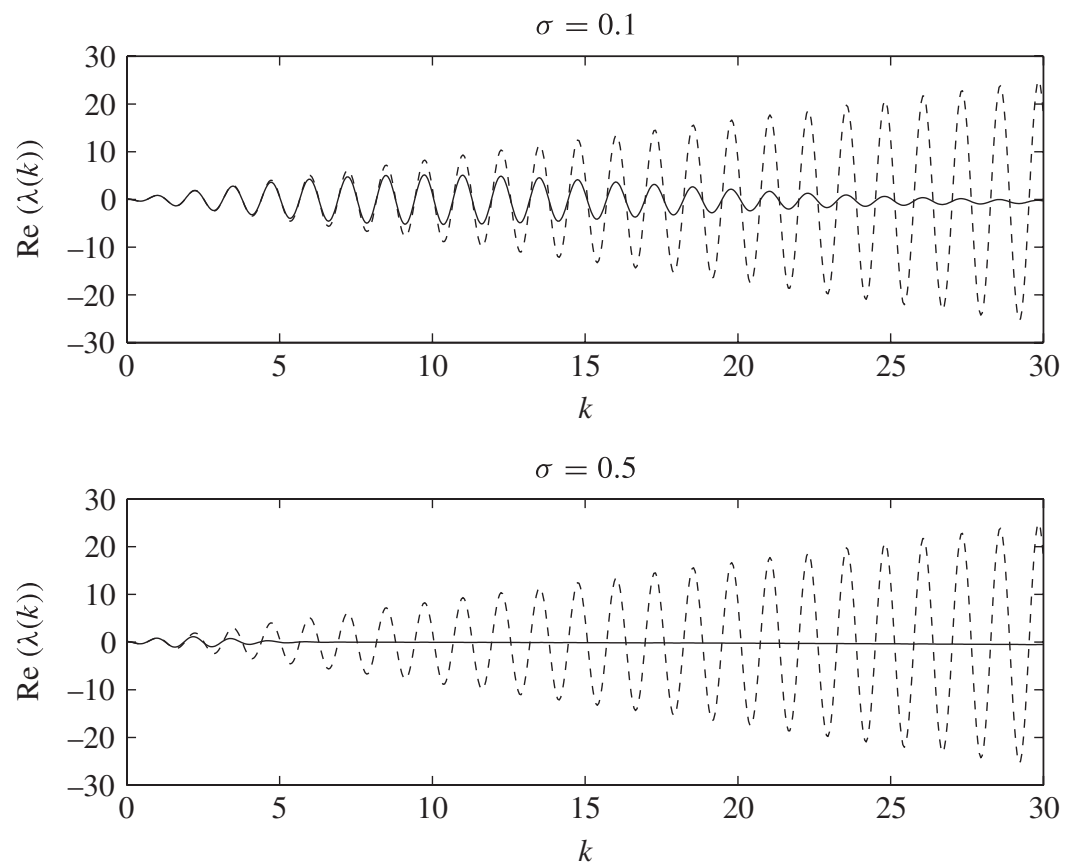

FIGURE 2. The real part of the eigenvalue $\lambda(k)$ against wavenumber $k$. The dashed curve is for a Dirac delta feeding kernel (4.2); the solid curve is for a Gaussian feeding kernel (4.1) with $\sigma=0.1$ (top panel) or $\sigma=0.5$ (bottom panel). Other parameter values: $\beta=5, K=0.2, \eta=0.1, \alpha \approx 1.3$ (determined by (3.3)).

gradual spreading of the body masses within a cohort [12]. Indeed, retaining secondorder terms in the jump-growth equation results in the addition of a diffusive term to the McKendrick-von Foerster equation that enhances stability of the steady state [13].

It has been shown in this paper that the steady state of the jump-growth model, corresponding to the well-known power-law relationship between body mass and abundance density described by equation (1.1), is always unstable in the case where a predator only feeds on prey items that are a fixed proportion of its own body mass. This result is significant because it highlights the importance of diet breadth in allowing a stable size spectrum. This is in keeping with previous stability results [13, 24], but is the first time that instability has been demonstrated analytically.

The results in this paper have been obtained for a system that spans an infinite range of body mass. Restriction to the more realistic case where there is a finite upper limit to body mass requires a more numerical approach [24]. However, the main conclusion of this study should apply regardless of the range of body masses permitted. This is because of the finding that eigenvalues with positive real part occur for arbitrarily large wavenumbers, that is, perturbations whose wavelength with respect to log body mass are arbitrarily short. This is consistent with numerical studies on finite body mass ranges [21], which have shown that the steady state is unstable when the diet breadth $\sigma$ is sufficiently small. 
Although it has been the focus of this study, diet breadth is not the only factor in the model that influences stability. For instance, increasing either the mean PPMR $\beta$ or the predator volume search rate exponent $\alpha$ can cause the steady state to lose stability [24].

The model considered here does not incorporate reproduction by mature adults. This is in keeping with many models, which have assumed a constant abundance density of organisms below a certain minimum size [6, 21], have assumed simple, zerogrowth dynamics for the abundance density of these organisms [4], or have considered an infinite range of body sizes [2]. More recently, models that include reproduction have been developed $[3,7,15,27]$. The question of stability of equilibrium states in these models remains largely unexplored.

\section{Acknowledgements}

This research was supported by RSNZ Marsden Fund grant number 08-UOC-034. The author thanks Samik Datta, Gustav Delius and Richard Law for illuminating discussions.

\section{References}

[1] S. Allesina and M. Pascual, "Network structure, predator-prey modules, and stability in large food webs", Theor. Ecol. 1 (2008) 55-64; doi:10.1007/s12080-007-0007-8.

[2] K. H. Andersen and J. E. Beyer, "A symptotic size determines species abundance in the marine size spectrum”, Am. Nat. 168 (2006) 54-61; doi:10.1086/504849.

[3] K. H. Andersen and M. Pedersen, "Damped trophic cascades driven by fishing in model marine ecosystems”, Proc. Roy. Soc. Lond. B 277 (2010) 795-802; doi:10.1098/rspb.2009.1512.

[4] E. Benoît and M.-J. Rochet, "A continuous model of biomass size spectra governed by predation and the effects of fishing on them", J. Theor. Biol. 226 (2004) 9-21; doi:10.1016/S0022-5193(03)00290-X.

[5] J. L. Blanchard, "The dynamics of size-structured ecosystems", Ph. D. Thesis, University of York, 2008.

[6] J. L. Blanchard, S. Jennings, R. Law, M. D. Castle, P. McCloghrie, M.-J. Rochet and E. Benoît, "How does abundance scale with body size in coupled size-structured food webs?", J. Anim. Ecol. 78 (2009) 270-280; doi:10.1111/j.1365-2656.2008.01466.x.

[7] J. L. Blanchard, R. Law, M. D. Castle and S. Jennings, "Coupled energy pathways and the resilience of size-structured food webs", Theor. Ecol. 4 (2011) 289-300; doi:10.1007/s12080-010-0078-9.

[8] P. R. Boudreau and L. M. Dickie, "Biomass spectra of aquatic ecosystems in relation to fisheries yield”, Can. J. Fish. Aqua. Sci. 49 (1992) 1528-1538; doi:10.1139/f92-169.

[9] J. Camacho and R. V. Solé, "Scaling in ecological size spectra", Europhys. Lett. 55 (2001) 774-780; doi:0.1209/epl/i2001-00347-0.

[10] J. A. Capitán and G. W. Delius, "Scale-invariant model of marine population dynamics", Phys. Rev. E 81 (2010) 061901; doi:10.1103/PhysRevE.81.061901.

[11] J. E. Cohen, S. L. Pimm, P. Yodzis and J. Saldaña, "Body sizes of animal predators and animal prey in food webs", J. Anim. Ecol. 62 (1993) 67-78; doi:10.2307/5483.

[12] S. Datta, G. W. Delius and R. Law, "A jump-growth model for predator-prey dynamics: derivation and application to marine ecosystems", Bull. Math. Biol. 72 (2010) 1361-1382; doi:10.1007/s11538-009-9496-5. Corrected version: http://arxiv.org/abs/0812.4968.

[13] S. Datta, G. W. Delius, R. Law and M. J. Plank, "A stability analysis of the power-law steady state of marine size spectra", J. Math. Biol. 63 (2011) 779-799; doi:10.1007/s00285-010-0387-z.

[14] T. Fenchel, "Intrinsic rate of natural increase: the relationship with body size", Oecolog. 14 (1974) 317-326; doi:10.1007/BF00384576. 
[15] M. Hartvig, K. H. Andersen and J. E. Beyer, "Food web framework for size-structured populations", J. Theor. Biol. 272 (2011) 113-122; doi:10.1016/j.jtbi.2010.12.006.

[16] M. R. Heath, "Size spectrum dynamics and the planktonic ecosystem of Loch Linnhe", ICES J. Mar. Sci. 52 (1995) 627-642; doi:10.1016/1054-3139(95)80077-8.

[17] S. Jennings and S. Mackinson, "Abundance-body mass relationships in size-structured food webs”, Ecol. Lett. 6 (2003) 971-974; doi:10.1046/j.1461-0248.2003.00529.x.

[18] S. Jennings, J. K. Pinnegar, N. V. C. Polunin and T. W. Boon, "Weak cross-species relationships between body size and trophic level belie powerful size-based trophic structuring in fish communities", J. Anim. Ecol. 70 (2001) 934-944; doi:10.1046/j.0021-8790.2001.00552.x.

[19] S. Jennings, K. J. Warr and S. Mackinson, "Use of size-based production and stable isotope analyses to predict trophic transfer efficiencies and predator-prey body mass ratios in food webs", Mar. Ecol. Prog. Ser. 240 (2002) 11-20; doi:10.3354/meps240011.

[20] S. R. Kerr and L. M. Dickie, The biomass spectrum: a predator-prey theory of aquatic production (Columbia University Press, New York, 2001).

[21] R. Law, M. J. Plank, A. James and J. L. Blanchard, "Size-spectra dynamics from stochastic predation and growth of individuals", Ecol. 90 (2009) 802-811; doi:10.1890/07-1900.1.

[22] O. Maury, B. Faugeras, Y.-J. Shin, C. Poggiale, T. B. Ari and F. Marsac, "Modelling environmental effects on the size-structured energy flow through marine ecosystems. Part 1: The model", Prog. Oceanog. 74 (2007) 479-499; doi:10.1016/j.pocean.2007.05.002.

[23] A. G. McKendrick, "Applications of mathematics to medical problems", Proc. Edinburgh Math. Soc. 40 (1926) 98-130.

[24] M. J. Plank and R. Law, "Ecological drivers of stability and instability in marine ecosystems". Theor. Ecol., to appear; doi:10.1007/s12080-01.

[25] T. Platt and K. Denman, "The structure of pelagic marine ecosystems", J. Conseil International pour l'Exploration de la Mer 173 (1978) 60-65.

[26] J. G. Pope, J. C. Rice, N. Daan, S. Jennings and H. Gislason, "Modelling an exploited marine fish community with 15 parameters-results from a simple size-based model", ICES J. Mar. Sci. 63 (2006) 1029-1044; doi:10.1016/j.icesjms.2006.04.015.

[27] M.-J. Rochet and E. Benoît, "Fishing destabilizes the biomass flow in the marine size spectrum", Proc. Roy. Soc. Lond. B 279 (2012) 284-292; doi:10.1098/rspb.2011.0893.

[28] E. San Martin, X. Irigoien, R. P. Harris, Á. López-Urrutia, M. Z. Zubkov and J. L. Heywood, "Variation in the transfer of energy in marine plankton along a productivity gradient in the Atlantic Ocean", Limnol. Oceanog. 51 (2006) 2084-3091; doi:10.4319/lo.2006.51.5.2084.

[29] F. S. Scharf, F. Juanes and R. A. Rountree, "Predator size-prey size relationships of marine fish predators: interspecific variation and effects of ontogeny and body size on trophic-niche breadth", Mar. Ecol. Prog. Ser. 208 (2000) 229-248; doi:10.3354/meps208229.

[30] R. W. Sheldon and T. Parsons, "A continuous size spectrum for particulate matter in the sea", J. Fish. Res. Board Canada 24 (1967) 909-915; doi:10.1139/f67-081.

[31] R. W. Sheldon, A. Prakash and W. H. Sutcliffe, "The size distribution of particles in the ocean", Limnol. Oceanog. 17 (1972) 327-340; doi:10.4319/lo.1972.17.3.0327.

[32] R. W. Sheldon, W. H. Sutcliffe and M. A. Paranjape, "Structure of pelagic food chain and relationship between plankton and fish production", J. Fish. Res. Board Canada 34 (1977) 2344-2353; doi:10.1139/f77-314.

[33] W. Silvert and T. Platt, "Energy flux in the pelagic ecosystem: a time-dependent equation", Limnol. Oceanog. 23 (1978) 813-816; doi:10.4319/lo.1978.23.4.0813.

[34] D. W. Stephens and J. R. Krebs, Foraging theory (Princeton University Press, Princeton, NJ, 1986).

[35] E. Ursin, "On the prey size preferences of cod and dab", Meddelelser fra Danmarks Fiskeri- og Havunders $\phi$ gelser 7 (1973) 85-98.

[36] H. von Foerster, "Some remarks on changing populations", in: The kinetics of cellular proliferation (ed. J. F. Stohlman), (Grune and Stratton, New York, 1959) 382-407.

[37] D. M. Ware, "Bioenergetics of pelagic fish: theoretical change in swimming speed and ration with body size”, J. Fish. Res. Board Canada 35 (1978) 220-228; doi:10.1139/f78-036. 